\title{
Parallelized and Vectorized Tracking Using Kalman Filters with CMS Detector Geometry and Events
} \author{
Würthwein ${ }^{1}$, and Avi Yagil $^{1}$ \\ ${ }^{1}$ UC San Diego, La Jolla, CA, USA 92093 \\ ${ }^{2}$ Princeton University, Princeton, NJ, USA 08544 \\ ${ }^{3}$ Cornell University, Ithaca, NY, USA 14853 \\ ${ }^{4}$ Fermilab, Batavia, IL, USA 60510-5011 \\ ${ }^{5}$ University of Oregon, Eugene, OR, USA 97403
}

Giuseppe Cerati ${ }^{4}$, Peter Elmer ${ }^{2}$, Brian Gravelle ${ }^{5}$, Matti Kortelainen ${ }^{4}$, Vyacheslav Krutelyov ${ }^{1}$, Steven Lantz ${ }^{3}$, Matthieu Lefebvre ${ }^{2}$, Mario Masciovecchio ${ }^{1}$, Kevin McDermott ${ }^{3}$, Boyana Norris $^{5}$, Allison Reinsvold Hall ${ }^{4}$, Daniel Riley ${ }^{3}$, Matevž Tadel ${ }^{1, *}$, Peter Wittich ${ }^{3}$, Frank

\begin{abstract}
The High-Luminosity Large Hadron Collider at CERN will be characterized by greater pileup of events and higher occupancy, making the track reconstruction even more computationally demanding. Existing algorithms at the LHC are based on Kalman filter techniques with proven excellent physics performance under a variety of conditions. Starting in 2014, we have been developing Kalman-filter-based methods for track finding and fitting adapted for many-core SIMD processors that are becoming dominant in high-performance systems.

This paper summarizes the latest extensions to our software that allow it to run on the realistic CMS-2017 tracker geometry using CMSSW-generated events, including pileup. The reconstructed tracks can be validated against either the CMSSW simulation that generated the detector hits, or the CMSSW reconstruction of the tracks. In general, the code's computational performance has continued to improve while the above capabilities were being added. We demonstrate that the present Kalman filter implementation is able to reconstruct events with comparable physics performance to CMSSW, while providing generally better computational performance. Further plans for advancing the software are discussed.
\end{abstract}

\section{Introduction}

Over the past few years, plans for the High-Luminosity Large Hadron Collider upgrade project, and the accompanying tenfold leap in luminosity, have made it clear that a significant research and development effort is required towards the 2020 to 2025 timeframe to meet the increased complexity and computational requirements of the track finding algorithms. The expected increase in event complexity, coupled with the technological changes that continue to drive interest in multi/many-core processors, have motivated the community to explore radically different algorithms and computing architectures to address the anticipated issues [1]. Our approach, however, has been to focus on the traditional, well-known,

*e-mail: mtadel@ucsd.edu 
and well-understood Kalman Filter (KF) method [2], to see how far KF-based tracking can be pushed in this new environment. To that end we have been developing the mkFit framework, designed from the ground up for performance, that is better suited to utilize the types of parallelism available in contemporary general-purpose computing hardware. The ultimate goal of the project is to reach physics performance on par with the standard Compact Muon Solenoid (CMS) tracking [3] while achieving a significant reduction in processing time.

All things considered, a fully-integrated, parallelized and vectorized implementation of a KF-based tracking application provides a strong reference point for the evaluation of more exotic types of solutions, both in terms of computational as well as physics performance. Indeed, new solutions of any type will have to demonstrate superior performance in both of these respects to operate successfully in environments with moderate time constraints such as high-level trigger applications and offline reconstruction.

The project was started in 2014 with the development of a "Matriplex" matrix operation library optimized for simultaneous vectorized processing of sets of small matrices. From this basis, the initial implementation of vectorized KF fitting was demonstrated on a simplified barrel-only detector [4].

The next stage was the implementation of track finding using the above technology and the same simplified geometry [5]. Simple multithreading was implemented by partitioning tracks in up to $21 \eta$ bins and using OpenMP parallel constructs. Physics performance was adequate (95\% efficiency and correct $\chi^{2}$ distribution of tracks and pulls of the track parameters), but the achieved parallelization speedups were a bit disappointing ( 2 for vectorization and $\mathrm{x} 10$ for multithreading on Intel KNC), indicating the need to decrease the fraction of nonvectorizable code and implement a better work partitioning scheme. To this end, processing of track candidates on each layer was optimized to reduce the number of instantiations of Track objects by selecting only the hits giving the best extensions to tracks based on their $\chi^{2}$ score before doing the final Kalman updates [6].

OpenMP was replaced by Intel Thread Building Blocks (TBB) to increase flexibility as well as to be in compliance with the CMS code base [7]. Further, to avoid imbalances in $\eta$ regions and to provide more workload tasks for the many available cores, support for processing of multiple events in parallel was added. This allowed the individual tasks to remain relatively large while still being able to fill up all available hardware threads [8].

Significant effort has been put into porting of mkFit to run on GPGPUs using CUDA [9]. Fitting and track finding have been ported for the barrel-only simplified detector. Performance results for track finding were disappointing with mkFit only being able to use about $4 \%$ of the available GPU processing power. Nevertheless, Matriplex is observed to outperform standard small-matrix multiplication packages for GPUs. We are currently in the process of quantifying performance plateaus reachable for KF-like operations as a function of problem size, problem segmentation, and arithmetic intensity with the intention of identifying architectural limitations to running KF-based track finding on GPGPUs.

Beginnning in 2015, mkFit was extended incrementally to handle realistic detector geometries with barrel and endcap sections. This required implementation of the KF and propagation equations for the endcap case, as well as a consolidated steering code that was able to handle both barrel and endcap cases. Finally, a general detector description mechanism was implemented to support arbitrary detector geometries.

Currently, mkFit is able to run on CMS-2017 geometry with reasonable physics and computational performance. Ongoing work is focusing on improving the physics performance through fine-tuning of hit and track selection algorithms. Post-processing of found tracks and duplicate track removal still needs to be implemented or may be delegated to algorithms in CMS software. 
This paper focuses on recent developments required to fully support realistic geometry of the CMS detector and to process simulated CMS data with up to 70 minimum bias $p p$ collisions superimposed over the signal $t \bar{t}$ events. Generalized geometry handling is described in section 2 and physics and computational performance are presented in sections 3 and 4.

\section{Handling of CMS geometry and events}

\subsection{Geometry and Detector description}

Geometry in mkFit is described as a vector of LayerInfo structures that contain the physical dimensions of a layer, hit search windows, and parameters and flags relevant for track finding. This includes information about layer detector type, stereo/mono layers, and an optional hole in detector coverage as needed for the CMS endcap detectors (this could be extended for even more general acceptance handling).

For track finding, steering parameters need to be defined for every tracking region. So far, it has been sufficient to consider only five distinct $\eta$ regions (barrel, $+z /-z$ transition, and $+z /-z$ endcap) but the concept could be used also to separate regions by $p_{T}$ or by tracking iteration. The steering parameters contain, most importantly, a vector of LayerControl structures that hold the layer indices (mapping into the LayerInfo vector) that need to be traversed during track finding. Additionally, it contains layer parameters and flags that are specific for this tracking region, such as tags for layers that are possible seeding layers or layers to be considered only during backward fitting. This allows the track finding algorithm to be completely agnostic of the detector structure: it simply follows the layer propagation plan in the steering parameters and executes operations in accordance with the control flags in LayerControl and LayerInfo structures.

Geometry and steering parameter setup is implemented as a plugin that populates the inmemory data structures with the required information. With this functionality, we are able to support both a simple geometry used for development and CMS-2017 geometry with all detector-specific information existing only in the plugin code. For the CMS-2017 geometry, we include the effects of multiple scattering and energy loss by defining two-dimensional arrays for the radiation and interaction lengths that are quickly indexed in $r-z$. These constants are taken from CMS simulation for the amount of material a particle would traverse propagating from module to module. mkFit supports usage of both constant and parameterized magnetic field; either type of field can be selected each time propagation is required in the code.

\subsection{Handling and processing of CMS events}

When processing CMS events mkFit relies on hit and seed data to be provided externally. In the standalone case (where mkFit operates independently of CMSSW), mkFit reads these data from a binary file created by a converter application. Additionally, the binary file can also contain vectors of simulated tracks and reconstructed tracks as found by standard CMS tracking, for later use in the validation of mkFit's performance.

Before passing seeds to mkFit for track finding, the seed collection is "cleaned" by removing multiple instances of seeds that are most likely based on hits belonging to the same outgoing particle. The cleaning algorithm uses the identity of hits and fitted seed parameters $p_{T}, \eta$, and $\varphi$ to eliminate duplicate seeds and is tuned so as to not cause any drop in track finding efficiency for high pile-up events. The duplicate seeds arise due to detector module overlaps that are rather significant, especially in the endcaps, where the modules on the same blade of a disk overlap considerably. 
In principle, seed cleaning could be performed as a final step in the seed finding algorithm; however, due to the way standard track finding works in CMSSW, this was not deemed necessary. CMSSW processes seeds one by one and when a track candidate is found, its hits are tagged as used. A seed is rejected if all its hits have already been used by a track candidate found earlier. The technique relies on CMSSW backward propagation to find additional hits on the seeding layers, and reduces the duplicate seeds in the first step of their consideration to a negligible level. This is not possible in mkFit where we process up to $32 \times N_{\text {threads }}$ seeds in parallel and, as we try to group seeds that are close in $\eta$ and $\varphi$ to maximize memory cache reuse of hit data, this could lead to significant waste of processing slots.

As already mentioned, we have recently started the process of including mkFit in standard CMS software distribution. mkFit is used as an external software package with a dedicated CMS processing module running within the CMS framework. This module packages the input data (seeds and hits) in format expected by mkFit, and provides high-level configuration and steering of mkFit execution. When an event is processed, it copies resulting tracks back into CMS format. This mode of inclusion allows mkFit code to remain independent of CMS particularities and overhead as well as allows us to perform development and testing in a more lightweight environment.

\section{Physics performance}

This section presents current basic physics performance plots for mkFit running on CMS2017 geometry with a CMSSW simulated sample of $5000 t \bar{t}$ events, each of which has been superimposed with a mean of 70 minimum-bias $p p$ collisions. Constant magnetic field of 3.8 T has been used. We are showing results corresponding to the CMS initial tracking iteration where seeds are required to have 4 hits all coming from distinct inner pixel layers and be compatible with the beam spot constraint. We show equivalent results from CMSSW using the same set of input seeds.

While these results show the actual performance of mkFit, they are preliminary in the sense that we know further work is necessary to make a fair comparison between CMSSW and mkFit initial iteration tracking:

- mkFit's hit selection windows, candidate scoring criteria, and final track quality criteria have not yet been tuned for optimal performance.

- Cleaning and merging of the final track collection have not yet been implemented in mkFit. This includes removal of duplicate tracks due to multiple seeds per particle.

- To ensure a fair comparison of efficiency, the same final track selection criteria and postprocessing need to be applied for both algorithms. CMSSW intentionally uses stronger requirements in initial iteration, relying on later iterations to pick up less likely track candidates.

Track finding efficiency versus $p_{T}$ and $\eta$ for mkFit and CMSSW are shown in figure 1. mkFit's performance is essentially equivalent to that of CMSSW for $p_{T}>0.9 \mathrm{GeV} / \mathrm{c}$, the standard CMS cut for detailed efficiency studies. Below that, mkFit's inefficiency is largest in the transition region and noticeable in the endcaps. We believe that tuning of hit selection windows and candidate scoring criteria can help us achieve efficiencies comparable to CMSSW for all tracking regions, down to $p_{T}=450 \mathrm{MeV} / \mathrm{c}$.

Figure 2 shows mkFit's duplicate track rates versus $p_{T}$ and $\eta$. CMSSW's duplicate rates are 0 . Duplicate rate of mkFit is significant for all values of $p_{T}$. In $\eta$, it is below $5 \%$ level in the barrel and rises sharply when tracks start entering the endcap disks. The duplicate rate distribution is exactly the same as for the artificial 10-muon events, showing that the duplicate 

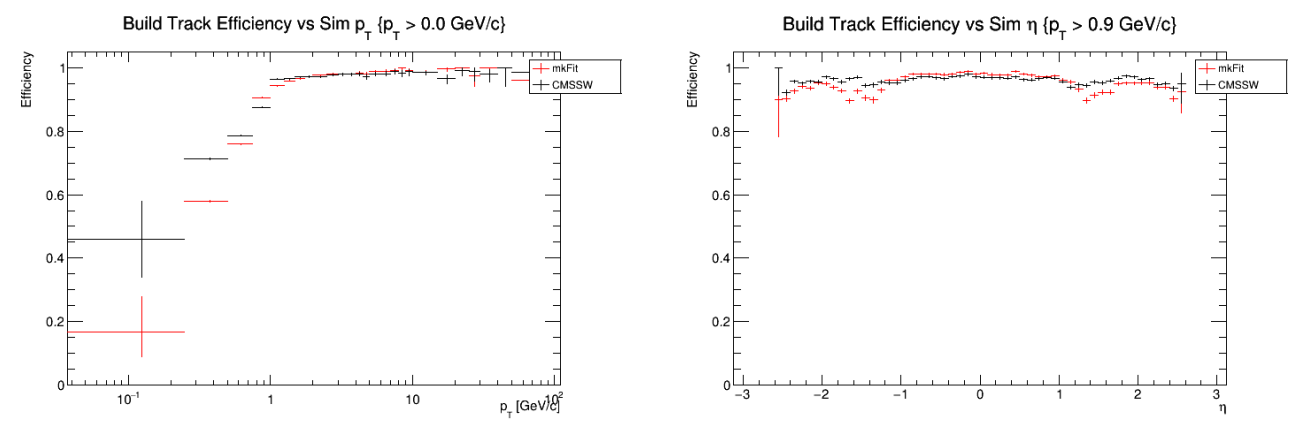

Figure 1. Efficiency versus $p_{T}$ (left) and efficiency versus $\eta$ for tracks with $p_{t}>0.9 \mathrm{GeV} / \mathrm{c}$, our target $p_{T}$ limit for CMS HLT operation (right).

rate is entirely due to duplicate seeds arising from module overlaps in the seeding layers and the absence of a duplicate track removal procedure in mkFit (see section 2.2).
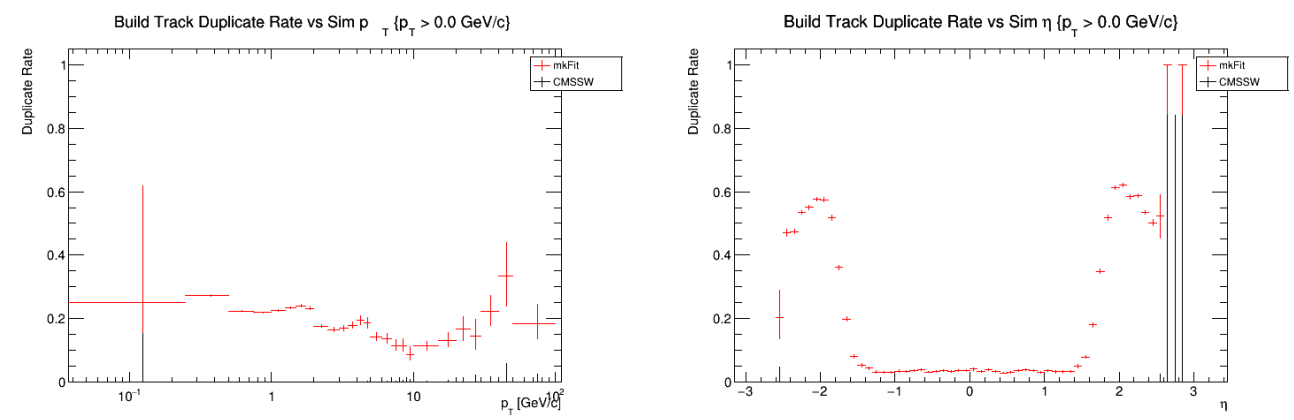

Figure 2. Duplicate track rate versus $p_{T}$ (left) and $\eta$ (right).

As already mentioned, further work is required to make a more detailed assessment of mkFit's performance. However, with mkFit being available within the CMSSW, all quality assurance and validation tools developed for CMS tracking are available for more detailed studies and debugging.

\section{Computational performance}

Computational benchmarks are shown for our main development platforms:

- KNL - Knights Landing - 64 cores: Intel Xeon Phi CPU 7210 @1.30GHz

- SKL-SP - Skylake Gold - 2 sockets x 16 cores: Intel Xeon Gold 6130 CPU @ 2.10GHz

While the Turbo Boost feature is turned off on all our development machines, SKL-SP processor cores feature different frequency characteristics depending on how many cores are in use and which vector instruction set is being used on them. KNL can also vary frequency to a lesser extent when AVX-512 code is being executed.

Results presented in this section were obtained using a subset of the events with the configuration described in the introduction to section 3. The Intel icc compiler was used to compile the code. mkFit uses Intel Thread Building Blocks (TBB) for multithreading. 


\subsection{Single event performance of core track finding}

To assess the performance of the track finding algorithm alone, we run a dedicated benchmark measuring the track-finding time for a sufficient number of events $\left(20 \times \mathrm{N}_{\text {threads }}\right)$ without including the time needed to pre-process the hits and seeds, or to post-process the track candidates. This allows us to focus on the most relevant part of our code and to sideline the more administrative tasks that might, in a production system, be performed outside of mkFit itself.

First, we show the speedup as a function of the Matriplex width which effectively controls how many slots in the vector registers are used. The results are shown in figure 3 . On both platforms the highest speedup is obtained with AVX-512 vectorization (SIMD width of 16 floats) and is 2.9 for SKL-SP and 3.3 for KNL. If we assume that vectorized code obtains the full speedup given by the Matriplex vector width, and that overall speedup is impaired only by unvectorized (serial) code sections, then Amdahl's Law implies that the code must be executing vector instructions at least $72 \%$ of the time (based on the final speedup of 3 for vector width 16).
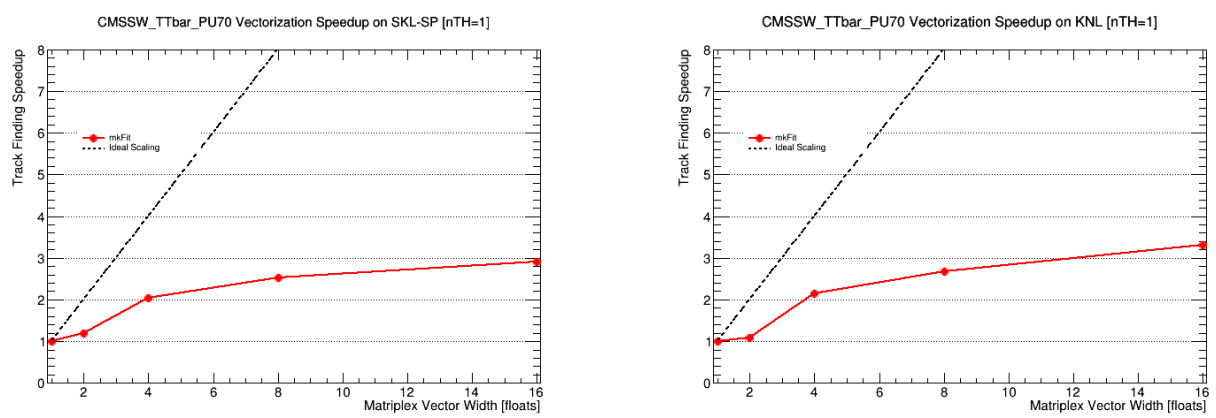

Figure 3. Vectorization speedup as a function of used vector width for Skylake (left) and Knights Landing (right) processors.

Figure 4 shows speedup as a function of the number of threads TBB is configured to use. Note that events are processed sequentially and all parallelism happens during the processing of seeds belonging to the same event. Beyond 32 threads the standard work chunk of 32 seeds per task gets progressively reduced down to 4 seeds per task at 256 threads. See [7] for details about how multithreading is implemented in mkFit. SKL-SP shows good scaling up to 8 threads and KNL up to 32 threads. For KNL the effect of reduction of the standard work chunk can be observed in continuation of scaling toward higher number of threads, up to about 164 threads.

\subsection{Full processing with multiple concurrent events in flight}

To assess the scaling behavior of the full event processing chain as it would run in CMSSW which can process several events concurrently, we implemented support for multiple concurrent events in flight in mkFit as well [8]. Technically, this is achieved by using the TBB parallel_for construct for the event loop itself and retaining all intra-event parallelism described before. This balances out the tail effects present in event-by-event processing and allows the tasks themselves to be larger, thus reducing the multithreading overhead. As before, the number of events processed for each test was 20 times the number of configured threads. 

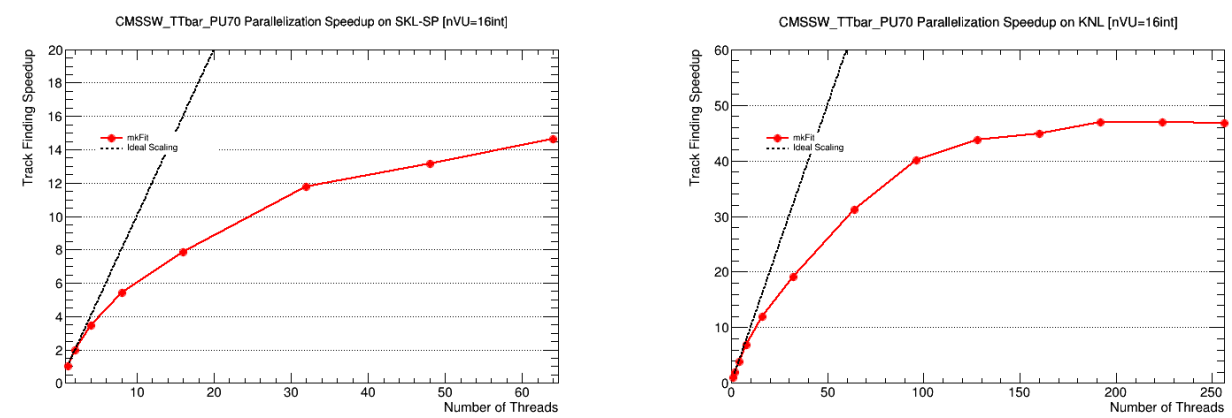

Figure 4. Multithreading speedup as a function of used number of threads for Skylake (left) and Knights Landing (right) processors.

Scaling behavior for multiple events in flight is shown in figure 5. Many of the administrative tasks related to pre-processing of hits and seeds have not yet been fully optimized or vectorized. One can see the effect of those by comparing results for one event in flight with corresponding result in the previous section, figure 4. SKL-SP shows best scaling with 16 events in flight; hyperthreading provides additional 30\% speedup when going from 32 to 64 running threads. For KNL, having 32 events in flight offers the best performance; up to 64 threads the same performance is also achieved by 16 events in flight. Having more than 32 events in flight is not helpful, possibly due to the fact that in KNL a given memory reference can only be "owned" by 1 of 32 tiles in the layout of cores. KNL shows no gain in using more than 128 threads, i.e., hyperthreading does not yield any additional speedup.
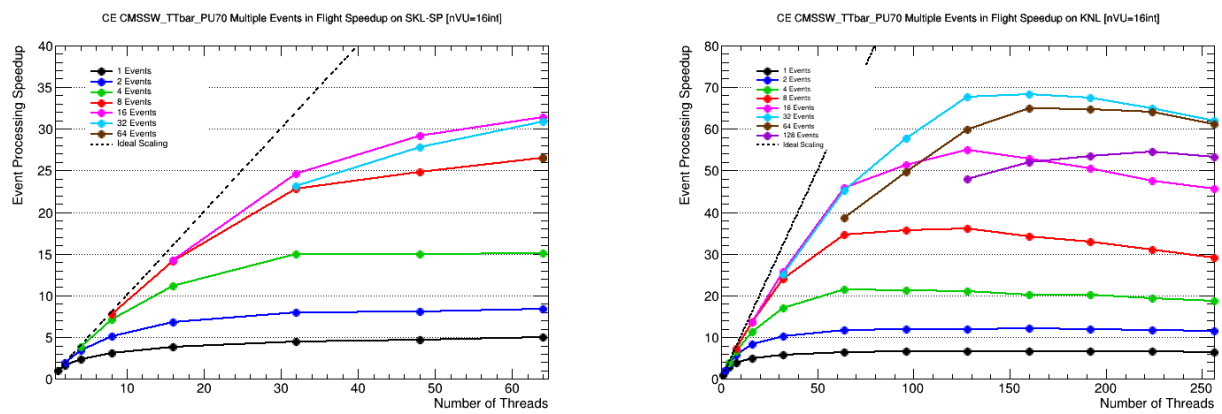

Figure 5. Multithreading speedup for different numbers of concurrent events in flight, as a function of used number of threads for Skylake (left), Knights Landing (right).

\subsection{Estimated performance of mkFit at CMS HLT}

In the CMS HLT, due to its processing time constraints, tracking is run only for a subset of all the input events. On the other hand, running on all events $(100 \mathrm{kHz}$ rate $)$ a version of the tracking similar to the one used offline would allow better event selection, cleaner physics data sets and thus better utilization of storage and CPU resources after data is already taken. Comparing CMSSW and standalone mkFit single-threaded performance of the initial offline tracking iteration, one finds that mkFit runs about 10-times faster than CMSSW offline tracking. Correspondingly, measured mkFit full-node event processing rates for the expected LHC Run 3 pileup of 70 are 115 events/s for KNL and 250 events/s for SKL-SP. Thus, to 
process events at CMS HLT at the expected $100 \mathrm{kHz}$ rate, one would need an equivalent of 400 32-core Skylake machines for track reconstruction alone. Note that this is below the current size of the CMS HLT cluster.

These results are to be considered as very preliminary. In fact, while on one side we believe the current version of the mkFit code can be further optimized, running in the actual HLT configuration within CMSSW requires more work to mitigate overheads due to data preparation (local reconstruction) and data conversions.

\section{Conclusion}

Following developments required to support complex, realistic detector geometries, mkFit is now in the position to demonstrate its potential for use in real-world reconstruction scenarios. Preliminary results show that mkFit exhibits physics performance on par with existing, traditional KF tracking algorithms while retaining a significant boost in computational performance. It also shows the potential to make efficient use of many-core architectures with few concurrent processes.

Ongoing work is focusing on finishing the tuning of track finding algorithm parameters and implementing the missing final post-processing of tracks. Integration with CMSSW is proceeding in parallel with the goal of integration in the CMS HLT test-bed system for Run 3 of the LHC.

\section{Acknowledgments}

This work is supported by the U.S. National Science Foundation, under the grants PHY1520969, PHY-1521042, PHY-1520942 and PHY-1624356, and by the U.S. Department of Energy, Office of Science, Office of Advanced Scientific Computing Research, Scientific Discovery through Advanced Computing (SciDAC) program.

\section{References}

[1] See, for example, the presentations and proceedings of the "Connecting the Dots 2018" workshop, https://indico.cern.ch/event/658267/overview

[2] Rudolf Fruhwirt, Nucl. Instrum. Meth. A262, 440-450 (1987)

[3] S. Chatrchyan et al. [CMS Collaboration], JINST 9, no. 10, P10009 (2014)

[4] Giuseppe Cerati et al., J. Phys.: Conf. Ser. 608, 012057 (2015)

[5] Giuseppe Cerati et al., J. Phys.: Conf. Ser. 664, 072008 (2015)

[6] Giuseppe Cerati et al., EPJ Web of Conferences 127, 00010 (2016)

[7] Giuseppe Cerati et al., J. Phys.: Conf. Ser. 898, 042051 (2017)

[8] Giuseppe Cerati et al., J. Phys.: Conf. Ser. 1085, 042016 (2018)

[9] Giuseppe Cerati et al., EPJ Web of Conferences 150, 00006 (2017) 